\title{
Desvantagem vocal em cantores populares
}

\author{
Voice disadvantage in popular singers
}

Camila Santos Sales ${ }^{1}$ (]) Silvana Pereira da Silva ${ }^{1}$ (), Adriane Mesquita de Medeiros ${ }^{1}$

\begin{abstract}
RESUMO
Objetivo: verificar a associação das características sociodemográficas, ocupacionais, de estilo de vida e saúde com a desvantagem vocal de cantores populares. Métodos: estudo observacional transversal com amostra de conveniência, composta por cantores populares. A coleta de dados foi realizada por meio de questionário com 21 perguntas, além do protocolo de Índice de Desvantagem para o Canto Moderno (IDCM). Foram utilizados os testes de Mann Whitney e Kruskal Wallis. Resultados: a amostra foi composta por 57 cantores, predominantemente do sexo masculino e com idade entre 30 e 39 anos. Houve significância estatística entre o escore total do IDCM e as variáveis: presença de mais de um cantor intercalando as músicas nas apresentações, menor tempo de profissão, não realização do desaquecimento vocal e autopercepção da voz falada como razoável. Conclusão: maior desvantagem vocal foi verificada em cantores mais novos na profissão, que não intercalam as músicas com outro cantor, não desaquecem a voz e percebem a voz falada como razoável.
\end{abstract}

Palavras-chave: Voz; Canto; Qualidade de vida; Saúde do trabalhador; Fonoaudiologia

\begin{abstract}
Purpose: Verify the association of sociodemographic, occupational, lifestyle and health characteristics with the vocal disadvantage of popular singers. Methods: Observational cross-sectional study with convenience sample composed of popular singers. The data collection was performed through a questionnaire with more than 21 questions, in addition to the protocol Modern Singing Handicap Index - MSHI. The Mann Whitney and Kruskal Wallis test were used. Results: The sample consisted of 57 singers, predominantly male, and the majority with age between 30 and 39 years. There was statistical significance between the total MSHI score and the variables: presence of more than one singer interposing the songs in the presentations, less time of profession, not realization of vocal quenching and self-perception of spoken voice as reasonable. Conclusion: Greater vocal disadvantage was verified in younger singers in the profession, who don't intercalate the songs with another singer, don't slow down the voice and perceive the spoken voice as reasonable.
\end{abstract}

Keywords: Voice; Singing; Quality of life; Occupational health; Speech therapy

Trabalho realizado na Universidade Federal de Minas Gerais - UFMG - Belo Horizonte (MG), Brasil.

${ }^{1}$ Universidade Federal de Minas Gerais - UFMG - Belo Horizonte (MG), Brasil.

Conflito de interesses: Não.

Contribuição dos autores: CSS realizou a coleta dos dados, a redação do manuscrito e a aprovação da versão final; SPS realizou a coleta dos dados, a redação do manuscrito e a aprovação da versão final; AMM participou da concepção do estudo, análise dos dados, orientou todas as etapas do trabalho e redação do manuscrito e aprovação da versão final.

Financiamento: Nada a declarar.

Autor correspondente: Silvana Pereira da Silva. E-mail: silvana_silva_3@hotmail.com

Recebido: Julho 31, 2018; Aceito: Junho 17, 2019 


\section{INTRODUÇÃO}

A voz é o principal recurso de trabalho do cantor. Estudo de revisão ${ }^{(1)}$ identificou elevada prevalência de distúrbios vocais autorrelatados entre cantores, principalmente entre os cantores populares, quando comparados aos clássicos. Em comparação a indivíduos com baixa demanda de voz, o cantor pode ter alterações vocais com mais frequência ${ }^{(2,3)}$.

O termo "canto popular" tem sido utilizado para diferenciar do canto clássico ou erudito. A música não clássica pode ser melhor denominada de música comercial contemporânea ${ }^{(4)}$ para descrever genericamente diversos estilos de música, como música popular brasileira (MPB), roque, samba, pop, pagode, gospel, sertanejo, entre outros.

Determinados tipos de desvio vocal no canto popular podem ser percebidos como uma alteração, ou interpretados como uma performance mais original e própria, dentro do contexto da música comercial contemporânea $^{(5)}$. $\mathrm{O}$ impacto do problema de voz no cantor pode se manifestar pela percepção de desvantagem no canto, ou seja, pelas dificuldades em manter a qualidade vocal, levando ao comprometimento da carreira ${ }^{(6)}$.

A alta demanda vocal dessa população apresenta diferentes graus de exigências e requintes, que podem interferir de maneiras distintas no exercício da profissão. No canto popular, a falta de treinamento formal e técnica de canto podem contribuir para o desenvolvimento distúrbios de voz e tal relação precisa ser melhor investigada ${ }^{(1)}$.

O conhecimento sobre a saúde vocal, para ser completo, deve analisar a perspectiva que o indivíduo tem em relação a sua qualidade de vida, mensurando o quanto esta pode ser afetada frente ao problema de $\operatorname{voz}^{(7)}$. A autopercepção pode ser considerada como um indicador para o estado de saúde dos cantores.

$\mathrm{Na}$ área da voz, existe o protocolo Índice de Desvantagem para o Canto Moderno (IDCM) $)^{(5,8)}$, com o objetivo de determinar como o problema da voz afeta os diversos aspectos da qualidade de vida do cantor e utilizado no presente estudo. Pesquisa mostrou que o uso de protocolo de autoavaliação genérico identifica menor desvantagem vocal em cantores, quando comparado aos protocolos específicos e direcionados para o canto, incluindo o IDCM. Sendo assim, para avaliar o impacto do problema de voz na vida dos cantores, faz-se necessário utilizar o protocolo específico para esse grupo ${ }^{(9)}$.

Pouco se sabe sobre os fatores que interferem na desvantagem vocal de cantores populares. A compreensão de como o comportamento e hábitos, as condições de saúde e trabalho desses cantores influenciam a percepção de desvantagem vocal é importante para as propostas de intervenções de promoção da saúde vocal e prevenção de distúrbios da voz, além do manejo terapêutico desse grupo.

O objetivo deste estudo foi verificar a associação das características sociodemográficas, comportamentais, ocupacionais e da saúde com a desvantagem vocal de cantores populares.

\section{MÉTODOS}

Realizou-se um estudo observacional transversal descritivo e analítico, com amostra de conveniência, composta por cantores populares de ambos os sexos. Foram incluídos os cantores populares que atuavam em bares, casas de show, festivais diurnos e noturnos. Foram excluídas bandas de música instrumental, cantores de conjuntos com menos de três integrantes e bandas com apenas um único estilo musical em seu repertório, na busca de comparar cantores com atividade de trabalho semelhante quanto à composição da equipe de trabalho. Tal critério também foi adotado para que se pudesse investigar um grupo de cantores populares, cuja demanda vocal incluísse uma diversidade de estilos musicais, como roque, MPB, pop, samba, pagode, gospel, sertanejo, entre outros. O projeto foi aprovado pelo Comitê de Ética em Pesquisa - COEP - UFMG, número do parecer 1.251.738.

Foi realizada, inicialmente, uma busca por meio de websites de conjuntos atuantes na região de Belo Horizonte e, então, os cantores foram contatados por telefone para serem informados sobre a pesquisa.

Estudo piloto foi realizado para verificar a compreensão dos cantores quanto às perguntas e o preenchimento do questionário, assim como identificar o tempo de resposta, que foi de, aproximadamente, 15 minutos.

Primeiramente, foi enviado o Termo de Consentimento Livre e Esclarecido (TCLE), via e-mail, e, após confirmação do interesse em participar, foram disponibilizados os questionários. Todos os participantes assinaram o TCLE. Dois instrumentos foram utilizados: um questionário construído pelos próprios pesquisadores, baseado na experiência clínica e em outro estudo com cantores $^{(10)}$ e o protocolo Índice de Desvantagem para o Canto Moderno - IDCM, validado por Fussi e Fuschini ${ }^{(8)}$.

O questionário elaborado pelos pesquisadores possuía 21 questões, com as seguintes variáveis explicativas: a) sociodemográficas: sexo (masculino e feminino), idade (19 a 29, 30 a 39, 40 a 49), canto como principal atividade de trabalho remunerada (não, sim), realização de outra atividade com o uso intenso da voz (não, sim); b) ocupacionais: tempo de profissão como cantor (variável contínua), carga horária de ensaio semanal (variável contínua), número de apresentações realizadas ao longo do mês (variável contínua), mais de um cantor intercalando as músicas durante as apresentações (não, sim), acompanhamento fonoaudiológico anterior ou atual para aperfeiçoar a voz cantada (não, sim), tratamento atual por problemas na voz (não, sim), realização de aquecimento vocal (nunca, sim raramente, sim às vezes, sim frequentemente) e desaquecimento vocal (nunca, sim raramente, sim às vezes, sim frequentemente); c) de saúde: percepção da voz falada (excelente, muito boa, boa, razoável, ruim), ingestão de água durante as apresentações (não, sim), quantidade de água por dia (mais de três litros, entre dois e três litros, menos de dois litros, nunca), sintomas de azia e má digestão durante ou após as apresentações (nunca, sim raramente, sim às vezes, sim frequentemente), ingestão de bebida alcoólica durante os shows (nunca, sim raramente, sim às vezes, sim frequentemente), prática de atividades físicas por semana (três ou mais vezes, uma até duas vezes, nenhuma vez), quadro de infecções de vias aéreas superiores (IVAS) (nunca, sim raramente, sim às vezes, sim frequentemente) e diagnóstico de refluxo gastroesofágico (RGE) (não, sim).

O protocolo $\operatorname{IDCM}^{(8)}$, traduzido para o Português ${ }^{(5)}$, foi aplicado e consta de 30 questões, distribuídas em três subescalas: a) a incapacidade, que está ligada ao aspecto funcional, relacionada ao impacto do problema da voz nas atividades profissionais; b) a desvantagem, relacionada ao impacto psicológico do problema da voz; c) o defeito, relativo ao aspecto orgânico, relacionado com a autopercepção das características da própria 
voz. O escore total do IDCM refere-se à somatória das três subescalas (incapacidade, desvantagem e defeito). As respostas foram assinaladas em uma escala de Likert de 5 pontos, de acordo com a frequência de ocorrência: 0 - nunca, 1- quase nunca, 2 - às vezes, 3 - quase sempre, 4 - sempre. Este protocolo apresenta três subescalas: incapacidade, desvantagem e defeito, cada uma com valor máximo de 40 pontos e o escore total é composto pela somatória dos anteriores, com um desvio máximo de 120 pontos (escore total). Quanto maior a pontuação, maior será a desvantagem percebida pelo indivíduo ${ }^{(11)}$.

Foi realizada uma análise descritiva dos dados, sendo que as variáveis categóricas foram representadas em porcentagem. As variáveis tempo de profissão como cantor (em anos), carga horária de ensaio (em horas), número de apresentações (últimos 30 dias), foram coletadas como contínuas e, depois, categorizadas para análise dos dados. As variáveis contínuas referentes ao IDCM foram representadas por medidas de tendência central.

Como variável resposta foi utilizado o escore total do IDCM, como variável contínua, para verificar a associação com as variáveis sociodemográficas, ocupacionais e de saúde, presentes no questionário.

Foi verificada a associação entre o escore total do IDCM e as demais variáveis explicativas, utilizando o teste de Mann Whitney (para comparação de dois grupos) e Kruskal Wallis (para comparação de 3 ou 4 grupos).

Foi considerado o nível de significância de 5\% (valor de $\mathrm{p}<0,05)$. Após a coleta de dados, os resultados obtidos foram organizados em um banco de dados criado em planilha Excel. As análises foram realizadas no software STATA(Stata Corporation, College Station, Texas), versão 12.0.

\section{RESULTADOS}

Participaram do estudo 57 cantores, a maioria do sexo masculino $(61,4 \%)$ e com faixa etária predominante entre 30 e 39 anos (40,4\%). Verificou-se que a maior média foi na subescala defeito (domínio orgânico) $(7,4, \pm 6,6)$ e a menor, na subescala desvantagem (domínio emocional) $(3,1, \pm 4,5)$. A média do escore total do IDCM foi de 16,6 $( \pm 15,4)$ (Tabela 1).

Verificou-se que a profissão de cantor como principal atividade de trabalho remunerada foi relatada por $56,1 \%$ dos participantes e a maioria deles exercia outra atividade com uso intenso da voz (52,6\%). Em relação ao tempo de canto, predominou os que possuíam entre 11 e 20 anos de experiência $(49,1 \%)$. Sobre a carga horária de ensaio de cada cantor, a maior parte foi de até 2 horas por semana $(47,4 \%)$ e $45,6 \%$ dos cantores haviam realizado até três apresentações nos últimos trinta dias (Tabela 2).

Durante as apresentações, a maioria dos cantores alternava as músicas, durante as apresentações, com outros cantores $(63,2 \%)$ e a maior parte respondeu que, frequentemente, utilizava o próprio equipamento para cantar (38,6\%). Aproximadamente $1 / 3$ dos participantes já havia feito acompanhamento fonoaudiológico para aperfeiçoar a voz cantada (29,8\%). Somente 3,5\% estavam fazendo tratamento por causa de problema de voz (Tabela 2).

Os cantores que, "às vezes" ou "frequentemente", realizavam exercícios de aquecimento vocal antes das apresentações representaram $82,5 \%$. Somente $19,3 \%$ relataram fazer o desaquecimento às vezes ou frequentemente, após as apresentações (Tabela 2). Houve associação estatisticamente significativa do escore total do IDCM com as seguintes variáveis: músicas intercaladas com outro cantor, menor tempo de carreira, não realizar desaquecimento vocal (Tabela 2).

Quanto à autopercepção da voz, a maior parte dos participantes relatou considerar a voz falada como boa $(38,6 \%)$. Os cantores que perceberam a voz falada como razoável apresentaram maior média do escore total do IDCM. A média do escore total foi indicando menor desvantagem vocal, à medida que a autopercepção vocal se tornava positiva (Tabela 3).

Foi frequente o relato dos cantores que ingeriam água durante os shows $(91,2 \%)$, mas em quantidade reduzida, ou seja, 52,6\% ingeriam menos de 2 litros por dia. Constatou-se, também, que $3,5 \%$ dos cantores nunca bebiam água e que $68,4 \%$ nunca ou raramente consumiam bebida alcoólica durante as apresentações. Mais de 2/3 dos participantes $(59,6 \%)$ relataram praticar atividades físicas regulares. Quanto à presença de quadro de IVAS, 66,7\% nunca ou raramente apresentaram algum sinal e/ou sintoma. Predominou o relato de 91,2\% dos cantores que nunca ou raramente sentiram azia e/ou má digestão, durante ou após as apresentações e 73,7\% não possuíam diagnóstico de RGE (Tabela 3).

\section{DISCUSSÃO}

Os cantores do presente estudo apresentaram elevada desvantagem vocal no canto popular. A média do escore total da desvantagem vocal entre os cantores participantes $(16,6)$ foi superior ao resultado de outro estudo com cantores clássicos $(8,6)$ e populares $(10,6)$, ambos sem queixa vocal ${ }^{(11)}$. Tal diferença era esperada, visto que os cantores desta pesquisa não foram selecionados segundo a presença, ou não, de queixa de voz. Além disso, os resultados mostraram que a maioria dos cantores exercia outra atividade remunerada, com uso intenso da voz, o que indica maior demanda vocal e maior chance de apresentar distúrbios vocais.

A escala de maior prejuízo foi relacionada à subescala defeito $(7,4)$, referente ao aspecto orgânico. Tais achados confirmam dados obtidos por outro estudo com cantores de coros universitários de música popular brasileira ${ }^{(5)}$, estudo com cantores de coros de igrejas católicas e evangélicas ${ }^{(12)} \mathrm{e}$ estudo com cantores populares, que comparou dois grupos, com e sem treinamento vocal $^{(2)}$. A diversidade de estilos de canto,

Tabela 1. Descrição das subescalas e escore total do Índice de Desvantagem para o Canto Moderno

\begin{tabular}{lccccc}
\hline \multicolumn{1}{c}{ Variável } & Média & Desvio padrão & Mediana & Mínimo & Máximo \\
\hline Incapacidade & 6,1 & 6,1 & 4 & 0 & 30 \\
Desvantagem & 3,1 & 4,5 & 2 & 0 & 21 \\
Defeito & 7,4 & 6,6 & 6 & 0 & 30 \\
Escore Total & 16,6 & 15,4 & 13 & 0 & 69 \\
\hline
\end{tabular}


Tabela 2. Descrição das características de trabalho dos cantores populares e associação com o escore total do Índice de Desvantagem para o Canto Moderno

\begin{tabular}{|c|c|c|c|c|c|c|c|}
\hline \multicolumn{2}{|l|}{ Variáveis } & \multirow{2}{*}{$\begin{array}{l}\mathrm{n} \\
35\end{array}$} & \multirow{2}{*}{$\frac{\%}{61,4}$} & \multirow{2}{*}{$\begin{array}{c}\text { Média } \\
17,7\end{array}$} & \multirow{2}{*}{$\begin{array}{c}\text { Desvio } \\
\text { padrão }\end{array}$} & \multirow{2}{*}{$\frac{\text { Mediana }}{13}$} & \multirow{2}{*}{$\begin{array}{c}\begin{array}{c}\text { Valor } \\
\text { de } p\end{array} \\
0,538\end{array}$} \\
\hline Sexo & Masculino & & & & & & \\
\hline & Feminino & 22 & 38,6 & 14,9 & 14,9 & 13 & \\
\hline \multirow[t]{3}{*}{ Faixa etária } & 19 a 29 & 22 & 38,6 & 19,2 & 17,6 & 15 & 0,477 \\
\hline & 30 a 39 & 23 & 40,3 & 15,4 & 13,4 & 13 & \\
\hline & 40 a 49 & 12 & 21,1 & 14,2 & 15,6 & 9,5 & \\
\hline \multirow[t]{2}{*}{ Canto como principal atividade remunerada } & Não & 25 & 43,9 & 16,5 & 14,8 & 13 & 0,803 \\
\hline & Sim & 32 & 56,1 & 16,7 & 16,1 & 13,5 & \\
\hline \multirow[t]{2}{*}{ Outra atividade vocal } & Não & 27 & 47,4 & 14,8 & 13,8 & 11 & 0,565 \\
\hline & $\operatorname{Sim}$ & 30 & 52,6 & 18,2 & 16,9 & 15 & \\
\hline \multirow[t]{3}{*}{ Tempo de profissão (em anos) } & 0 a 10 & 23 & 40,4 & 20,9 & 14,6 & 19 & $0.014^{*}$ \\
\hline & 11 a 20 & 28 & 49,1 & 14,7 & 15,5 & 11,5 & \\
\hline & 21 a 30 & 6 & 10,5 & 9 & 16,3 & 2,5 & \\
\hline \multirow[t]{3}{*}{ Carga horária de ensaio (em horas) } & 0 a 2 & 27 & 47,4 & 13,1 & 11,4 & 10 & 0,403 \\
\hline & 3 a 5 & 18 & 31,6 & 19,9 & 19,6 & 14,5 & \\
\hline & 6 a 25 & 12 & 21,0 & 19,4 & 16 & 15 & \\
\hline \multirow[t]{3}{*}{ Número de apresentações (últimos 30 dias) } & 1 a 3 & 26 & 45,6 & 17 & 18 & 12 & 0,864 \\
\hline & 4 a 7 & 17 & 29,8 & 15,4 & 13,2 & 11 & \\
\hline & 8 a 25 & 14 & 24,6 & 17,2 & 13,7 & 17,5 & \\
\hline \multirow[t]{2}{*}{ Alternância com outro cantor } & $\operatorname{Sim}$ & 36 & 63,2 & 13,3 & 14,1 & 10 & $0.013^{* *}$ \\
\hline & Não & 21 & 36,8 & 22,2 & 16,4 & 20 & \\
\hline \multirow[t]{4}{*}{ Equipamento próprio } & Nunca & 10 & 17,6 & 10,8 & 9,2 & 9 & 0,328 \\
\hline & Sim, raramente & 8 & 14,0 & 12 & 10,4 & 13 & \\
\hline & Sim, às vezes & 17 & 29,8 & 16,8 & 15,6 & 12 & \\
\hline & Sim, frequentemente & 22 & 38,6 & 20,8 & 18,3 & 16,5 & \\
\hline \multirow{2}{*}{$\begin{array}{l}\text { Acompanhamento fonoaudiológico para voz } \\
\text { cantada }\end{array}$} & Não & 40 & 70,2 & 16,6 & 15,7 & 11,5 & 0,619 \\
\hline & Sim & 17 & 29,8 & 16,6 & 15,4 & 15 & \\
\hline \multirow[t]{2}{*}{ Tratamento atual para distúrbio de voz } & Não & 55 & 96,5 & 15,7 & 14 & 12 & 0,193 \\
\hline & Sim & 2 & 3,5 & 42 & 38,2 & 42 & \\
\hline \multirow[t]{4}{*}{ Aquecimento vocal } & Nunca & 4 & 7,0 & 8,5 & 9,9 & 6,5 & 0,572 \\
\hline & Sim, raramente & 6 & 10,5 & 19 & 25 & 12,5 & \\
\hline & Sim, às vezes & 22 & 38,6 & 18,4 & 14,6 & 14 & \\
\hline & Sim, frequentemente & 25 & 43,9 & 15,7 & 14,5 & 15 & \\
\hline \multirow[t]{4}{*}{ Desaquecimento vocal } & Nunca & 24 & 42,1 & 18,4 & 19,1 & 14 & $0.037^{*}$ \\
\hline & Sim, raramente & 22 & 38,6 & 18,9 & 13 & 16 & \\
\hline & Sim, às vezes & 7 & 12,3 & 4,6 & 4,5 & 3 & \\
\hline & Sim, frequentemente & 4 & 7,0 & 14,3 & 5 & 13 & \\
\hline
\end{tabular}

*Teste de Kruskal Wallis significativo a 5\%; ${ }^{* *} T e s t e$ de Mann Whitney significativo a $5 \%$

Legenda: $n=$ número de sujeitos

educação vocal e demanda ocupacional parecem contribuir para a variabilidade na prevalência de alteração de voz autorrelatada ${ }^{(1)}$. A desvantagem vocal em cantores pode ser interpretada como falta de conhecimento técnico, grande demanda vocal e curta experiência no canto ${ }^{(5,13,14)}$.

Os resultados do presente estudo confirmaram a maior desvantagem vocal em cantores com o menor tempo de experiência no canto popular. Um curto tempo na carreira, como cantor popular, pode predispor a um menor tempo de consciência vocal e treinamento formal para lidar com a demanda de trabalho. Um estudo ${ }^{(15)}$ encontrou que, quanto maior o tempo de experiência no canto, menor a desvantagem vocal mensurada pelo protocolo Índice de Desvantagem Vocal no Canto Clássico (IDVCC). Outros estudos verificaram que a correlação entre o tempo de profissão, o escore total ${ }^{(16)}$ e as três subescalas do $\operatorname{IDCM}^{(9)}$ foi negativa, ou seja, há maior desvantagem vocal entre cantores com curto período de carreira. Por outro lado, outros estudos não encontraram associação estatisticamente significativa na correlação entre o tempo de carreira e a desvantagem vocal dos cantores líricos e populares ${ }^{(17)} \mathrm{e}$ em cantores de igreja ${ }^{(12)}$.

Os resultados deste estudo também mostraram que cantores que não intercalam as músicas com outro cantor, durante os shows, apresentam maior desvantagem vocal, em relação aos cantores que alternam. Tais resultados são semelhantes a outro estudo, que comparou o comportamento vocal de uma performance vocal individual com uma performance vocal compartilhada com outros cantores ${ }^{(18)}$. Tal comportamento permite que o cantor realize um repouso vocal parcial durante as apresentações, o que demonstrou favorecer a melhor saúde vocal.

No presente estudo, também foi percebido que os cantores que não tinham o hábito de desaquecer a voz, após as apresentações 
Tabela 3. Descrição das características do estilo de vida e saúde dos cantores populares e associação com o escore total do Índice de Desvantagem para o Canto Moderno

\begin{tabular}{|c|c|c|c|c|c|c|c|}
\hline \multicolumn{2}{|l|}{ Variáveis } & \multirow{2}{*}{$\begin{array}{c}\mathbf{n} \\
14\end{array}$} & \multirow{2}{*}{$\begin{array}{c}\% \\
24,5\end{array}$} & \multirow{2}{*}{$\begin{array}{c}\text { Média } \\
8,6\end{array}$} & \multirow{2}{*}{$\begin{array}{c}\text { Desvio } \\
\text { padrão }\end{array}$} & \multirow{2}{*}{$\begin{array}{c}\text { Mediana } \\
4\end{array}$} & \multirow{2}{*}{$\begin{array}{c}\begin{array}{c}\text { Valor } \\
\text { de } p\end{array} \\
0.009\end{array}$} \\
\hline Percepção da voz falada & Excelente & & & & & & \\
\hline & Muito boa & 18 & 31,6 & 15 & 10,7 & 14 & \\
\hline & Boa & 22 & 38,6 & 18,6 & 12,2 & 16,5 & \\
\hline & Razoável & 3 & 5,3 & 48,7 & 33,5 & 67 & \\
\hline \multirow[t]{2}{*}{ Ingestão de água durante as apresentações } & Não & 5 & 8,8 & 13 & 3 & 13 & 0,933 \\
\hline & $\operatorname{Sim}$ & 52 & 91,2 & 16,9 & 16,1 & 13,5 & \\
\hline \multirow[t]{4}{*}{ Quantidade água (em litros) por dia } & $>3$ & 6 & 10,5 & 15,8 & 16,4 & 10,5 & 0,394 \\
\hline & De 2 a 3 & 19 & 33,3 & 14,1 & 14,4 & 11 & \\
\hline & $<2$ & 30 & 52,6 & 18,9 & 16,3 & 15 & \\
\hline & Nunca & 2 & 3,6 & 8 & 11,3 & 8 & \\
\hline \multirow[t]{4}{*}{ Ingestão de bebida alcoólica } & Nunca & 32 & 56,2 & 16,2 & 15,7 & 12 & 0,336 \\
\hline & Sim, raramente & 7 & 12,3 & 11,4 & 15,2 & 4 & \\
\hline & Sim, às vezes & 10 & 17,5 & 19,3 & 19 & 18,5 & \\
\hline & $\begin{array}{l}\text { Sim, frequente } \\
\text { mente }\end{array}$ & 8 & 14,0 & 19,3 & 10,4 & 17,5 & \\
\hline \multirow[t]{3}{*}{ Prática de atividade física (por semana) } & $\geq 3$ & 21 & 36,8 & 17,6 & 19,2 & 13 & 0,87 \\
\hline & 1 a 2 & 13 & 22,8 & 17,4 & 14,2 & 12 & \\
\hline & Nenhuma & 23 & 40,4 & 15,3 & 12,6 & 15 & \\
\hline \multirow[t]{4}{*}{ Infecção vias aéreas superiores } & Nunca & 13 & 22,8 & 12,8 & 14,7 & 6 & 0,226 \\
\hline & Sim, raramente & 25 & 43,8 & 15,3 & 14 & 12 & \\
\hline & Sim, às vezes & 16 & 28,1 & 19,6 & 18,3 & 17 & \\
\hline & Sim, frequentemente & 3 & 5,3 & 27,3 & 13,7 & 25 & \\
\hline \multirow[t]{2}{*}{ Refluxo gastroesofágico } & Não & 42 & 73,7 & 15,9 & 15,8 & 11,5 & 0,404 \\
\hline & Sim & 15 & 26,3 & 18,6 & 14,8 & 15 & \\
\hline \multirow[t]{4}{*}{ Sintomas de Azia } & Nunca & 45 & 78,9 & 15,2 & 15 & 12 & 0,304 \\
\hline & Sim, raramente & 7 & 12,3 & 19,3 & 17,5 & 13 & \\
\hline & Sim, às vezes & 4 & 7,0 & 27 & 18,5 & 29 & \\
\hline & Sim, frequentemente & 1 & 1,8 & 20 & . & 20 & \\
\hline
\end{tabular}

*Teste de Kruskal Wallis significativo a $5 \%$

Legenda: $\mathrm{n}$ = número de sujeitos

musicais, apresentaram maior desvantagem vocal. Verificou-se que a média do escore total do IDCM foi menor entre os cantores que relataram desaquecer a voz às vezes, ou frequentemente. Outro estudo encontrou 21 cantores populares e 13 cantores líricos, que nunca realizaram o desaquecimento vocal ${ }^{(17)}$. Destaca-se que um elevado número de cantores populares participantes deste estudo realizou aquecimento vocal às vezes, ou frequentemente. Tanto o aquecimento, quanto o desaquecimento vocal são importantes, pois estes procedimentos podem dar condições para que os cantores consigam realizar os ajustes vocais, sem prejudicar a sua vida profissional ${ }^{(14,19,20)}$. O aquecimento e o desaquecimento vocal personalizado proporcionam ao cantor popular maior flexibilidade vocal, permitindo a exploração, de forma saudável, de suas marcas e identidade vocal ${ }^{(14)}$.

Os cantores que autoperceberam a voz falada como razoável, apresentaram maior desvantagem vocal no canto popular, quando comparados aos demais entrevistados com uma avaliação mais positiva da voz. A voz falada e a voz cantada têm realidades diferentes, mas tal resultado indica uma relação entre ambas. A voz falada ocorre de forma natural, inconsciente e, normalmente, o falante expõe o que pensa. A voz cantada, geralmente é consciente e é necessário aperfeiçoamento vocal, devido às necessidades dos diferentes estilos musicais, sendo a música popular considerada o estilo mais espontâneo e sem regras predeterminadas ${ }^{(21)}$.
Um estudo que objetivou avaliar parâmetros vocais relacionados à percepção da voz falada pelos cantores verificou que apenas um participante percebeu prejuízo na qualidade vocal. A percepção positiva da própria qualidade da voz falada diminui as possibilidades de relato de alterações referentes a questões orgânicas na voz cantada ${ }^{(22)}$. Em um estudo com coralistas, houve significância estatística entre a ausência de sintomas na voz falada e a boa percepção da qualidade da voz na fala, pelos cantores ${ }^{(23)}$. A percepção do distúrbio vocal na voz falada de cantores populares pode indicar dificuldades e prejuízos também na voz cantada, sendo que ambas precisam ser consideradas e avaliadas durante a atuação fonoaudiológica(24).

É importante conhecer os comportamentos e hábitos vocais dos cantores populares, para que seja possível um trabalho fonoaudiológico de orientação e conscientização sobre os cuidados com a voz e, em consequência, conservação da saúde vocal. A maioria dos cantores relatou boa saúde geral, quanto a presença de IVAS, sintomas de azia e diagnóstico de RGE. Autores mencionaram a vibração irregular da mucosa das pregas vocais como um dos comprometimentos ocasionados pelo refluxo ácido em pacientes com RGE, o que justificaria a disfonia apresentada por estes pacientes ${ }^{(25,26)}$. Infecções nas vias aéreas superiores podem contribuir ou aumentar o mau uso vocal, uma vez, que as alterações fazem com que o uso da voz se dê em condições inapropriadas, ou seja, na presença 
de mucosas ressecadas, edemas e irritação do trato vocal ${ }^{(27)}$. Apesar das consequências de tais morbidades sobre a voz, a presença destas entre os cantores não influenciou a percepção sobre a desvantagem vocal no canto.

Hábitos saudáveis, como a prática regular de atividade física e a não ingestão de bebidas alcóolicas durante as apresentações foram relatos frequentes entre os participantes.

O presente estudo constatou que havia uma boa hidratação durante as apresentações com os cantores, porém, a quantidade diária de ingestão de água relatada pela maioria foi inferior a dois litros. Tal quantidade é considerada insuficiente, apesar da ausência de evidência científica sobre a quantidade de água ideal a ser consumida diariamente, para se manter a hidratação laríngea. Sabe-se que a laringe bem hidratada favorece a menor pressão de ar sobre as pregas vocais para iniciar a fonação, reduzindo o nível de pressão de fonação e a fadiga vocal. Além disso, o atrito entre as pregas vocais decorrente do pigarro e da tosse pode ser evitado ${ }^{(28)}$.

Apesar do número reduzido de participantes deste estudo e da impossibilidade de generalização dos resultados para população de cantores populares, os resultados trouxeram contribuições sobre a desvantagem vocal e sua relação com aspectos do trabalho e do treinamento técnico para o canto. Algumas atitudes, como intercalar músicas com outro cantor e realização de aquecimento e desaquecimento vocal, além de adequada hidratação diária, podem reduzir o impacto do problema de voz nas atividades profissionais dos cantores.

A intervenção fonoaudiológica pode minimizar as alterações e também tem caráter preventivo, ajudando, assim, os profissionais da voz a cuidarem do seu instrumento de trabalho ${ }^{(29)}$, o que leva a pensar na longevidade da carreira dos cantores, diminuindo os riscos de possíveis aparecimentos de alterações vocais ${ }^{(10)}$.

Espera-se que sejam desenvolvidas mais pesquisas, com intuito de investigar aspectos referentes ao canto popular, englobando não só a voz do cantor, como também as condições emocionais, sociais, de trabalho e hábitos de bem-estar vocal. O maior conhecimento a respeito da voz cantada e das peculiaridades de cada estilo musical é fundamental para a Fonoaudiologia, pois permitirá o desenvolvimento de conduta integral e especializada.

\section{CONCLUSÃO}

Cantores populares apresentaram desvantagem vocal no canto, principalmente no aspecto orgânico (subescala defeito). Maior desvantagem vocal foi verificada entre aqueles que não intercalam as músicas com outro cantor, durante as apresentações, aqueles com menor tempo de carreira, os que não desaquecem a voz e que autopercebem a voz falada como razoável.

Hábitos como aquecer a voz antes das apresentações e realizar o desaquecimento, aumentar o consumo de água durante o dia e intercalar as músicas com outro cantor são estratégias que podem minimizar a desvantagem vocal em cantores populares.

\section{REFERÊNCIAS}

1. Pestana PM, Vaz-Freitas S, Manso MC. Prevalence of voice disorders in singers: systematic review and meta-analysis. J Voice. 2017 Nov;31(6):722-7. http://dx.doi.org/10.1016/j.jvoice.2017.02.010. PMid:28342677.
2. Silva FF, Moreti F, Oliveira G, Behlau M. Effects of vocal rehabilitation on voice handicap of professional popular singers. Audiology Communic Res. 2014;19(2):194-201. http://dx.doi.org/10.1590/ S2317-64312014000200015.

3. Mota LAA, Santos CMB, Barbosa KMF, Nascimento JR No. Disfonia em cantores: revisão de literatura. ACTA ORL/Técnicas em Otorrinolaringologia. 2010;28(1):27-21.

4. LoVetri JL, Weekly EM. Contemporary commercial music (CCM) survey: who's teaching what in nonclassical music. J Voice. 2003 June;17(2):207-15. http://dx.doi.org/10.1016/S0892-1997(03)00004-3. PMid:12825653.

5. Moreti F, Rocha C, Borrego MCM, Behlau M. Voice handicap in singing: analysis of the Modern Singing Handicap Index - MSHI. Rev Soc Bras Fonoaudiol. 2011;16(2):146-51. http://dx.doi.org/10.1590/ S1516-80342011000200007.

6. Rosen CA, Murry T. Voice handicap index in singers. J Voice. 2000 Sep;14(3):370-7. http://dx.doi.org/10.1016/S0892-1997(00)80082-X. PMid:11021504.

7. Señaris González B, Núñez Batalla F, Corte Santos P, Suárez C No. Índice de Incapacidad Vocal: factores predictivos Factors predicting voice handicap index. Acta Otorrinolaringol Esp. 2006;57(2):101-8. http://dx.doi.org/10.1016/S0001-6519(06)78670-8.

8. Fussi F, Fuschini T. Foniatria artística: la presa in carico foniatricologopedica del cantante clássico e moderno. Audiologia \& Foniatria. 2008;13(1-2):4-28.

9. Paoliello K, Oliveira G, Behlau M. Singing voice handicap mapped by different self-assessment instruments. CoDAS. 2013;25(5):463-8. http://dx.doi.org/10.1590/S2317-17822013005000008.

10. Goulart BNG, Rocha JG, Chiari BM. Group speech-language pathology intervention in popular singers: prospective controlled study. J Soc Bras Fonoaudiol. 2012;24(1):7-18. http://dx.doi.org/10.1590/S217964912012000100004. PMid:22460367.

11. Moreti F, Ávila MEB, Rocha C, Borrego MCM, Oliveira G, Behlau M. Influence of complaints and singing style in singers voice handicap. J Soc Bras Fonoaudiol. 2012;24(3):296-300. http://dx.doi.org/10.1590/ S2179-64912012000300017. PMid:23128180.

12. Prestes T, Pereira EC, Bail DI, Dassie-Leite AP. Vocal handicap of church singers. Rev CEFAC. 2012;14(5):901-9. http://dx.doi. org/10.1590/S1516-18462012005000035.

13. Coelho A, Daroz I, Silvério K, Brasolotto A. Amateur choir singers: self-image, difficulties and symptoms of the singing voice. Rev CEFAC. 2013;15(2):436-43. http://dx.doi.org/10.1590/S151618462013000200021.

14. Behlau M, Moreti F, Pecoraro G. Customized vocal conditioning for singing profissional voice users - case report. Rev CEFAC. 2014;16(5):1713-22. http://dx.doi.org/10.1590/1982-021620147113.

15. Avila MEB, Oliveira G, Behlau M. Índice de desvantagem vocal no canto clássico (IDCC) em cantores eruditos. Pro-Fono. 2010;22(3):221-6. http://dx.doi.org/10.1590/S0104-56872010000300011. PMid:21103709.

16. Loiola-Barreiro CM, Silva MA. Vocal handicap index in popular and erudite professional singers. CoDAS. 2016;28(5):602-9. http://dx.doi. org/10.1590/2317-1782/20162015226. PMid:27849245.

17. Dassie-Leite AP, Duprat AC, Busch R. A comparison between vocal habits of lyric and popular singers. Rev CEFAC. 2011;13(1):123-31. http://dx.doi.org/10.1590/S1516-18462010005000118.

18. Zambão VR, Penteado RZ, Calçada MLM. Working conditions and professional voice use by ballroom band singers. Rev CEFAC. 2014;16(6):1909-18. 
19. Gonsalves A, Amin E, Behlau M. Overall voice and strain level analysis in rock singers. Pró-Fono Revista de Atualização Científica. 2010;22(3):195-200. http://dx.doi.org/10.1590/S0104-56872010000300007. PMid:21103705.

20. Mello EL, Andrada e Silva MA. The singer's body: stretch, relax or warm-up. Rev CEFAC. 2008;10(4):548-56. http://dx.doi.org/10.1590/ S1516-18462008000400015.

21. Zimmer V, Cielo CA, Ferreira FM. Vocal behavior of popular singers. Rev CEFAC. 2012;14(2):298-307. http://dx.doi.org/10.1590/S151618462011005000101 .

22. Silva EGF, Luna CLC. Perceptual vocal pattern analysis of singers from kitschy musical style in Recife. Rev CEFAC. 2009;11(3):457-64. http://dx.doi.org/10.1590/S1516-18462009000300013.

23. Ribeiro LR, Hanayama EM. Amateur choir: vocal profile. Rev CEFAC. 2005;7(2):252-66.

24. Rocha C, Moraes M, Behlau M. Pain in popular singers. J Soc Bras Fonoaudiol. 2012;24(4):374-80. http://dx.doi.org/10.1590/S217964912012000400014. PMid:23306689.
25. Zucato B, Behlau MS. Laryngopharyngeal Reflux Symptoms Index: relation with the main symptoms of gastroesophageal reflux, voice usage level and voice screening. Rev CEFAC. 2012;14(6):1197-203. http://dx.doi.org/10.1590/S1516-18462012005000078.

26. Vera LR, Fuess MCL. Vocal problems in kindergarten and primary school teachers: prevalence and risk factors. Rev Bras Otorrinolaringol. 2003;69(6):807-12.

27. Tavares JG, Silva EHAA. Theoretical considerations on the relationship between mouth breathing and dysphonia. Rev Soc Bras Fonoaudiol. 2008;13(4):405-10. http://dx.doi.org/10.1590/S1516-80342008000400017.

28. Van Wyk L, Cloete M, Hattingh D, van der Linde J, Geertsema S. The effect of hydration on the voice quality of future professional vocal performers. J Voice. 2017;31(1):111.e29-111.e36. http://dx.doi. org/10.1016/j.jvoice.2016.01.002.

29. Ribeiro VV, Santos AB, Bonki E, Prestes T, Dassie-Leite AP. Identification of vocal problems experienced by church singers. Rev CEFAC. 2012;14(1):90-6. http://dx.doi.org/10.1590/S151618462011005000055 . 